\title{
Prevalence of Tick-borne Pathogens in Co-grazed Dairy Bovines Differs by Region and Host-type in Tamil Nadu, India
}

\author{
Ponnudurai ${ }^{1 \#}$, Stephen Larcombe ${ }^{2^{*}}$, Velusamy $\mathbf{R}^{3}$, Rani $\mathbf{N}^{3}$, Kolte $\mathbf{S W}^{3}$, Rubinibala $\mathbf{B}^{4}$, Alagesan $\mathbf{A}^{4}$, Rekha $\mathbf{B}^{4}$ and Brian Shiels ${ }^{2}$ \\ ${ }^{1}$ Department of Veterinary Parasitology, Faculty of Tamil Nadu, Veterinary and Animal Sciences University, Chennai, India \\ ${ }^{2}$ Institute of Biodiversity, Animal Health and Comparative Medicine, University of Glasgow, UK \\ ${ }^{3}$ Department of Veterinary Parasitology, Faculty of Maharashtra, Animal and Fisheries Sciences University, Nagpur, India \\ ${ }^{4}$ Department of Veterinary Parasitology, Veterinary College and Research Institute, Namakkal, India
}

*Corresponding author: Stephen Larcombe, Institute of Biodiveristy, Animal Health and Comparative Medicine, University of Glasgow, UK, Tel: +44 (0)141 330 8098; Email: stephen.larcombe@glasgow.ac.uk

Received date: 21 April, 2017; Accepted date: 11 May, 2017; Published date: 20 May, 2017

Copyright: (c) 2017 Ponnudurai G, et al. This is an open-access article distributed under the terms of the Creative Commons Attribution License, which permits unrestricted use, distribution, and reproduction in any medium, provided the original author and source are credited.

\#-contributed equally to the work.

\begin{abstract}
In India, disease of cattle caused by tick-borne pathogens (TBP) constrains the rearing of high yielding Bos taurus dairy breeds that often develop clinical, fatal disease. Bos indicus and bufflao show resistance to TBP infection, and this has led to a policy of rearing crossbred indicus-taurus cattle. On dairy farms, co-grazing more resistant animals alongside crossbreeds could contribute positively (by removing potentially infective ticks) or negatively (by acting as a reservoir for infection). We investigated epidemiological factors that contribute to the prevalence of five tick-borne pathogens with links to milk-yield in co-grazed host types in dairy farms of two regions of Tamil Nadu, India; a region of high dairy production importance. A high prevalence of T. annulata and Anaplasma spp. was detected, but with lower prevalence in the Cauvery Delta than in the Northwestern zone. A strict host-type association with prevalence was observed: buffaloes had lower prevalence of TBP than cattle; and native breeds had a lower prevalence of Anaplasma spp. than crossbreeds. The results indicate that while susceptibility to becoming a carrier animal for TBP depends on exposure to ticks; aspects of resistance are determined by host type independent of tick exposure. There was no clear evidence that co-grazing, with more resistant host types, will provide a positive contribution (protective effect) to crossbreeds with greater milk productivity.
\end{abstract}

Keywords: Theileria annulata; Health; Anaplasma; Host breed type

\section{Introduction}

Tropical bovine theileriosis is a tick-borne disease (TBD) caused by Theileria annulata belonging to the phylum Apicomplexa. Globally around 250 million cattle are at risk from the disease. In India, the dairy industry is important (estimated 160 million metric tonnes in 2017), but the prevalence of cattle infected with tick-borne pathogens (TBP) can be high and constrains the rearing of high-yielding Bos taurus breeds. For this reason, milk in India is generally produced by less productive native cattle or buffalo, or by Bos indicus ${ }^{\star}$ Bos taurus cross-breed cattle [1] that have greater productivity than native breeds. However, even among crossbred cattle $T$. annulata can cause acute pathology while in native breeds and buffaloes infection manifests primarily as a subclinical form [2,3]. Economic loss due to tropical theileriosis in India is estimated to be US\$800 million [4] but this is likely to be an underestimate as substantial losses from subclicinal carrier infection are rarely captured [5]. A recent survey carried out in Maharashtra state, India showed that the fitness cost to apparently healthy animals with subclinical infection of tick-borne haemoparasites (Theileria and Babesia) were more pronounced in crossbreed than in native breed cattle [6]. Such losses are of importance in India, where a growing population relies on dairy products as a primary source of protein [1].

Currently, tick control in India is mainly achieved by treatment of animals with acaricide at a level that prevents, or reduces, tick challenge [7]. Environmental issues with acaricide use and development of resistance limits effectiveness [8], and novel strategies to control ticks and TBD are required. Such strategies will be informed through greater understanding of regional epidemiology of TBD. This includes identification of risk factors associated with infection and the influence of host (cattle vs buffalo) or breed (crossbreed vs native) type on becoming a carrier animal, and a source of infection to susceptible cattle. This information would be of particular value if particular reservoir host types differentially influence transmission efficiency to susceptible host types, as postulated for other tick-borne pathogens, e.g. Borrelia [9]. Thus, depending on the local level of tick challenge, if more resistant hosts acted to remove of a subsection of viable infective vectors, combined with a reduced probability of remaining as a carrier, the challenge to more susceptible and productive cattle may be reduced. To assess whether such a scenario could operate in India and have impact in designing optimal stocking practises, a greater understanding of host type in determining prevalence of TBP carrier status is needed.

Tamil Nadu is one of the top 10 regions of milk production in India, where a mixture of breed and host types are reared [3], with a higher proportion of cross-breeds in its dairy herds than other regions [1]. Episodes of tropical theileriosis are known to occur persistently in the central region of the state. This study deployed both blood smear and PCR diagnosis to determine the prevalence of infection with $T$. annulata, and other TBP in bovine hosts reared in two agro climatic zones of central Tamil Nadu. Prevalence data was then used to test for any extrinsic or intrinsic risk factors associated with carrier infection 
Citation: Ponnudurai G, Larcombe S, Velusamy R, Rani N, Kolte SW, et al. (2017) Prevalence of Tick-borne Pathogens in Co-grazed Dairy Bovines Differs by Region and Host-type in Tamil Nadu, India. J Adv Dairy Res 5: 177. doi:10.4172/2329-888X.1000177

Page 2 of 7

with emphasis on differences between different breed/host type and therefore, their potential to act as reservoirs for transmission to susceptible hosts.

\section{Materials and Methods}

\section{Study area}

Samples were collected from October, 2014 to March, 2015 from animals reared in two agro climatic zones viz., Cauvery delta zone and North Western zone located in the central part of Tamil Nadu, India (Figure 1). The climate of both of the sampling zones areas is hot and humid $\left(28.8^{\circ} \mathrm{C}\right.$ average temperature), with an annual rainfall of 764 $\mathrm{mm}$ and $860 \mathrm{~mm}$ in Cauvery delta and North western zone, respectively.

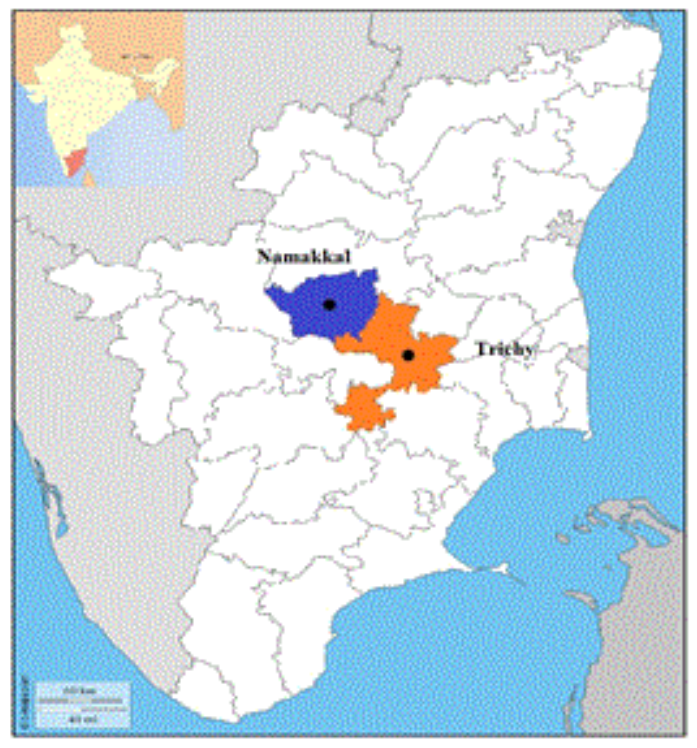

Figure 1: Map showing geographical location of sampling areas: Cauvery delta zone (Trichy district) and North Western zone (Namakkal district).

\section{Sample and data collection}

Blood samples were collected from 331 apparently healthy animals, which include crossbred cattle (123), native cattle (105) and buffaloes (103), from randomly selected working, productive dairy herds located in Musiri, Pavithram and Thottiyam Taluk of Trichy district (North Western Zone), and Alanganatham, Erumapatti and Varagur village areas of Namakkal district (Cauvery delta zone). In this area animals are co-grazed, although some native breeds (especially males) are housed separately as working animals. Blood was collected from the jugular vein of animals in vacutainer tubes coated with $0.5 \mathrm{M}$ EDTA. Blood smears were prepared with blood obtained by puncturing the ear vein. Data pertaining to breed, sex, and age were collected through questionnaires. We also thoroughly assessed Animals were thoroughly assessed by visual inspection for tick infestations. Though this involved whole body searches particular attention was paid to known predilection sites for individual species ie. Boophilus spp.: all over the body; Hyalomma spp.: udder, inguinal region, below the base of the tail, tip of the tail; Rhipicephalus haemaphysaloides. ear, eyelids and neck region; Haemaphysalis. in ear, and eyelids. Where animals were found to be infested, the ticks (all stages) were counted and collected in a vial containing 70 per cent ethanol. Ticks were identified using three different keys [10-12]. Ticks were then scored into the following categories: (1) <5 ticks; (2) 5-20 ticks; (3) 20-50 ticks; (4) >50 ticks. Age data was available in different levels of detail for each herd (based on breeding history, age in years or tooth eruption), To standardize this data and simplify analyses, all animals were assigned an age class: $<2$, $2-4$ and $>4$ year old.

\section{PCR screening}

Genomic DNA was extracted from whole blood using a commercial DNA extraction kit (Qiagen Blood and Tissue Kit, Chatsworth, CA, USA), according to the manufacturer's instruction; isolated DNA was stored at $-80^{\circ} \mathrm{C}$. A PCR assay targeting the $18 \mathrm{~s}$ rRNA gene was utilised for amplification of 5 different TBP based on conditions and primers, as described previously [6]. Specificity of the PCR was tested by direct sequencing two PCR products, and BLAST analysis of the amplicons. Each of the tests showed exclusive amplification of products from the intended species. Parasite DNA amplified at a species specific level represented: Theileria annulata, Therileria orientalis, Babesia bigemina, and Babesia bovis. Less specific primers were designed to amplify Anaplasma; +ve samples were scored as Anaplasma spp. for the results from this assay [6]. PCR amplification was performed in a final volume of $25 \mu \mathrm{l}$, with each reaction containing $4 \mu \mathrm{l}$ of DNA template, $4.5 \mu \mathrm{l}$ of nuclease free water, $12.5 \mu \mathrm{l}$ of $1.5 \mathrm{mM} \mathrm{Mgcl}_{2}$ (Taq 2X Master mix, Red Ampliqon) and $2 \mu \mathrm{l}$ of each primer at 10 pmol. Target amplification was carried out in a thermal cycler (Eppendorf, Germany) using the reaction profile described Junlong et al. [13], modified as follows: 35 cycles of three steps each, comprising initial denaturing at $95^{\circ} \mathrm{C}$ for 30 sec, primer annealing at $55^{\circ} \mathrm{C}$ for $30 \mathrm{sec}$ and product extension at $72^{\circ} \mathrm{C}$ for $30 \mathrm{sec}$. Amplified products were separated by electrophoresis on a $1 \%$ agarose gel using standard running conditions and visualized by ethidium bromide stain under UV using a Gel documentation system (BioRad, USA). Positive controls (DNA isolated from animals known to be infected with specific parasites by blood smear) and negative control (Distilled PCR-grade water) were used in every run. All reactions produced specific, single bands.

\section{Blood smear examination}

For all samples blood smears were generated to compare detectability across PCR and microsocopy techniques for T. annulata. Thin blood smears were prepared on glass slides, fixed in methanol for 40 minutes and stained with Giemsa stain ( 1 in 20 dilution in deionised $\mathrm{H}_{2} \mathrm{O}$ ) for 40 minutes, washed in $\mathrm{H}_{2} \mathrm{O}$ and examined microscopically under oil immersion. A minimum of 50-100 fields per smear were examined for detection of Theileria spp. based on morphology of the piroplasm stage.

\section{Statistical Analyses (Risk Factor Association)}

To produce estimates of the relative importance of different intrinsic and extrinsic factors influencing the prevalence of disease we used a mixed modelling approach (Generalized Linear Mixed Models (GLMM)) using the GLIMMIX procedure in SAS (v 3.1. SAS Inc, USA). Use of a GLMM approach allowed us to flexibly model nonnormally distributed response variables, accounting for fixed factors (Sex, Age Class, Host type and Tick scores), while including geographic location as a random effect (Block) to control for non-independence of hosts from the same location of origin [14]. Two models containing 
different response variables were constructed: the prevalence of Theileria annulata as a binary distributed response, and the prevalence of Anaplasma spp. as a binary distributed response. Other parasites were encountered at a frequency too low to be modelled statistically. To perform risk factor associations we included all fixed factors noted above in each model, and all two or three-way interactions between them. Non-significant interactions were removed until only statistically significant terms remained. Statistically significant factors that remained were considered to be potential (positive or negative) risk factors for the parasites in question. In some cases significant terms or interactions had more than two levels, or more than one significant fixed factor. In these cases, determining the biological relevance of the significant effect can be difficult. To ease interpretation we computed Least Squares Means (LS Means). LS Means are group means computed for some fixed factor of interest having controlled for further covariate/s (e.g. in our case, means of host type while holding geographic location constant), and LS means can be compared using tukey's t-tests to find out which groups (from factors with $>2$ levels) are significantly different from one another using GLIMMIX [14]. Further details on the direction (positive or negative) of each risk, and on construction of the models is provided in the results, as appropriate.

\section{Ethical Statement}

This study was carried out in strict accordance with the recommendations of the Veterinary Council of India and all work was overseen by their staff. Ethical approval was not required for performing animal experiments (Institutional Animal Ethics Committee of Tamil Nadu Veterinary and Animal Sciences University) as: 1 . the survey was conducted by government official veterinary physicians in village farms within their jurisdiction, 2. samples were taken as part of the standard course of veterinary inspection to determine presence of infections detrimental to animal health in India and 3. in India, ethical approval is not required for survey work conducted for the benefit of livestock welfare or improved farming practice, as in this case. No animals were housed or harmed as part of the survey, and every care was taken to minimise any suffering of the animals during the brief handling period.

\section{Results}

\section{Prevalence of infection and sensitivity of diagnostic techniques}

Initial analysis of blood samples was performed by microscopic analysis of blood smears for detection of Theileria spp. piroplasm forms. Of the 331 blood smears examined, piroplasms could be detected in 11 smears (3.3\%). All 11 positive results were obtained from crossbred cattle, thus none of samples from native cattle and buffaloes were scored as positive for Theileria spp. by microscopy.

The PCR generated clear evidence of specific amplification of the predicted size products, with $T$. annulata and Anaplasma spp. amplicons the most commonly detected. Specific amplification was validated by sequencing in a parallel study that used the same primers and PCR conditions: the top BLAST hits for sequenced PCR products matched the intended target [6]. Results of the PCR assay for $T$. annulata were initially correlated with location and host type: $76.2 \%$, $68.0 \%$ and $26.4 \%$ of crossbred cattle, native cattle and buffaloes, respectively from the North Western region were infected, and $67.1 \%$, $54.5 \%$ and $4.0 \%$ in crossbred cattle, native cattle and buffaloes respectively from the Cauvery delta region. These differences were explored in more detail using statistical approaches (see below). The results support previous studies demonstrating diagnosis of carrier animals is significantly more sensitive by PCR assay relative to blood smear $[15,16]$. All samples that scored as positive for Theileria spp. by blood smear gave a positive result for T. annulata in the PCR assay. The significantly lower level of prevalence in buffalo supports the blood smear data, while for native breeds this correlation was not obtained, as the level of infection by PCR was similar to that of the crossbreed animals. Taking the results of the two tests together it can be postulated that the buffalo sampled are either not challenged by infected ticks or are resistant to establishment of a carrier infection; while native breeds are more likely, relative to crossbreeds, to present a carrier state that is below the sensitivity threshold of diagnosis by blood smear. Using PCR we also detected a high prevalence of Anaplasma spp. $(\mathrm{n}=128$, see below), but very few cases of Babesia (B. bovis $\mathrm{n}=4$; B. bigemina $\mathrm{n}=1$ ).

\section{Tick infestations}

We constructed chi-square analyses on presence/absence tick data and found that while overall there was no difference in the prevalence of ongoing tick infestations between locations (Northwestern vs. Cauvery Delta: $\left.\mathrm{X}^{2}=0.009, \mathrm{p}=0.9\right)$ there was a significant effect of host type $\left(\mathrm{X}^{2}=25.98, \mathrm{p}<0.0001\right)$, with buffalo harbouring far fewer tick infestations than native or cross-breed cattle. To investigate in more detail, we constructed chi-square analyses using the presence/absence of different tick species. Four different tick species were detected on our study animals: Hyalomma anatolicum, Boophilus microplus, Haemaphysalis intermedia and Rhipicephalus spp. (Table 1). There was no difference between crossbreed and native cattle in the presence/ absence of any individual tick species. However, for Boophilus microplus and Haemaphysalis intermedia crossbreed cattle had significantly more ticks than buffalo $\left(\mathrm{X}^{2}=0.009, \mathrm{p}=0.9\right.$, cross breed vs buffalo tukey adjusted $\mathrm{p}<0.05)$ and for Hyaloma anatolicum crossbreed and native cattle had significantly more ticks than buffalo $\left(\mathrm{X}^{2}=0.009\right.$, $\mathrm{p}=0.9$ cattle vs buffalo tukey adjusted $\mathrm{p}<0.05$ ). Although, the overall difference in tick infestations between regions was not significant, there was a significant difference in some individual ticks species. There were greater numbers of both Haemaphysalis intermedia and Rhicephalus in the Northwestern zone than in the Cauvery Delta, although both were rare overall.

\section{Intrinsic and extrinsic factors influencing TBP prevalence}

Theileria annulata: Preliminary inspection of prevalence data from Theileria infected animals indicated a possible influence of geographic origin. Although principally we aimed to control for any impact of shared geographic origin in our models, it was considered interesting to test for a geographic effect. Therefore, before constructing full GLMM models with geographic origin as a random effect, we constructed more simple GLMs including breed, location and a location $^{*}$ breed interaction to investigate the statistical significance of location effects. Using this approach we found significant impact of both breed (see below) and location ( $F 1,316=9.04, p=0.0013)$. In general, prevalence was higher in North Western Zone than in the Cauvery Delta; moreover, although the interaction term breed ${ }^{\star}$ location was not significant, post-hoc comparisons using least squares means showed that overall differences in Theileria annulata prevalence between the locations were driven more by buffaloes $(26.4 \%$ vs. $4 \%$ : $\mathrm{t}=-2.74$, tukey adjusted $\mathrm{p}=0.0065)$ than by native $(68 \%$ vs $54.5 \%$ : $\mathrm{t}=-1.15$, tukey adjusted $\mathrm{p}=0.25)$ or cross breed $(76.2 \%$ vs $67.1 \%$ : 
Citation: Ponnudurai G, Larcombe S, Velusamy R, Rani N, Kolte SW, et al. (2017) Prevalence of Tick-borne Pathogens in Co-grazed Dairy Bovines Differs by Region and Host-type in Tamil Nadu, India. J Adv Dairy Res 5: 177. doi:10.4172/2329-888X.1000177

Page 4 of 7

$\mathrm{t}=-1.35$, tukey adjusted $\mathrm{p}=0.18$ ) cattle. This result demonstrates that extrinsic factors operating at even a small geographic scale can impact on the local epidemiology of Theileria infection, while highlighting the importance of controlling for geographic origin in further statistical tests.

\begin{tabular}{|c|c|c|c|c|c|c|c|c|}
\hline Region & Breed & $\mathrm{N}=$ & $\begin{array}{l}\text { No. on-going } \\
\text { tick infestations }\end{array}$ & $\begin{array}{l}\text { Hyalomma } \\
\text { anatolicum }\end{array}$ & $\begin{array}{l}\text { Boophilus } \\
\text { microplus }\end{array}$ & $\begin{array}{l}\text { Haemaphysalis } \\
\text { intermedia }\end{array}$ & $\begin{array}{l}\text { Rhipicephalus } \\
\text { spp. }\end{array}$ & Mixed infestation \\
\hline \multirow{4}{*}{$\begin{array}{l}\text { North } \\
\text { Western }\end{array}$} & Cross & 59 & 19 & 9 & 1 & 6 & 4 & 3 \\
\hline & Native & 50 & 14 & 8 & 3 & 3 & - & 1 \\
\hline & Buffalo & 53 & 5 & - & - & - & 5 & - \\
\hline & Total & 162 & 38 & 17 & 4 & 9 & 9 & 4 \\
\hline \multirow{4}{*}{$\begin{array}{l}\text { Cauvery } \\
\text { Delta }\end{array}$} & Cross & 64 & 22 & 13 & 8 & 1 & - & \\
\hline & Native & 55 & 15 & 12 & 2 & 1 & - & 1 \\
\hline & Buffalo & 50 & - & - & - & - & - & - \\
\hline & Total & 169 & 37 & 25 & 10 & 2 & - & 1 \\
\hline
\end{tabular}

Table 1: Counts of on-going tick infestations in different host types in Cauvery Delta and North Western Zone.

To investigate in more detail how intrinsic and extrinsic differences impact the epidemiology of $T$. annulata we constructed GLMM models testing for impacts of sex, age, host breed/type and tick infestation on parasite prevalence/carrier state.

\begin{tabular}{|l|l|l|l|}
\hline \multicolumn{1}{|c|}{ Parasite } & \multicolumn{1}{|c|}{ Fixed factor } & \multicolumn{1}{c|}{$\mathbf{F}_{\text {d.f }}$} & \multicolumn{1}{c|}{ P-value } \\
\hline \multirow{4}{*}{ T. annulata } & Sex & $0.25_{1,311}$ & 0.6198 \\
\cline { 2 - 5 } & Age class & $3.07_{2,314}$ & 0.047 \\
\cline { 2 - 5 } & Host type & $25.77_{2,314}$ & $<0.0001$ \\
\cline { 2 - 5 } & Tick infestation & $4.11_{1,314}$ & 0.044 \\
\hline \multirow{4}{*}{ Anaplasma spp. } & Sex & $4.55_{1,315}$ & 0.033 \\
\cline { 2 - 5 } & Age class & $0.68_{2,313}$ & 0.5 \\
\cline { 2 - 5 } & Host type & $8.38_{2,315}$ & 0.0003 \\
\cline { 2 - 5 } & Tick infestation & $12.01_{1,314}$ & 0.0006 \\
\hline
\end{tabular}

Table 2: Risk factor association outputs from GLMMs for T. annulata and Anaplasma spp. No statistically significant interactions were found, values of non-significant main effects provided at point of removal from model. (Model Intercepts: $T$ annulata: -0.66 +/- 0.57: Anaplasma: -0.32 +/- 1.3 Covariance parameter estimates for random effect location: T annulata: $0.3+/-0.47$ : Anaplasma: $3.329+/-4.78$ ).

We found no evidence for any statistically significant interactions; however, there was a significant impact of host type and host age (Table 2). Figure 2 shows that buffalo are significantly less likely than cross breed (LSmeans $\mathrm{t}=-6.95, \mathrm{p}<0.0001$ ) or native (LSmeans $\mathrm{t}=-4.78$, $\mathrm{p}<0.0001)$ cattle to carry T. annulata, and that native and cross breed cattle do not significantly differ in prevalence (LSmeans $t=-0.32$, $\mathrm{p}>0.75$ ). Overall, the prevalence of $T$. annulata increased with age across host types/breeds (Figure 3). Surprisingly, in the initial model tick score was not retained as a statistically significant fixed factor (F3, $312=1.73, \mathrm{p}=0.160$ ). However, the raw data was suggestive of an impact of tick infestation, in general, plus dividing the intensity score data across four different categories could abrogate the important question of whether tick exposure per se impacts on prevalence of carrier infection in different host types.

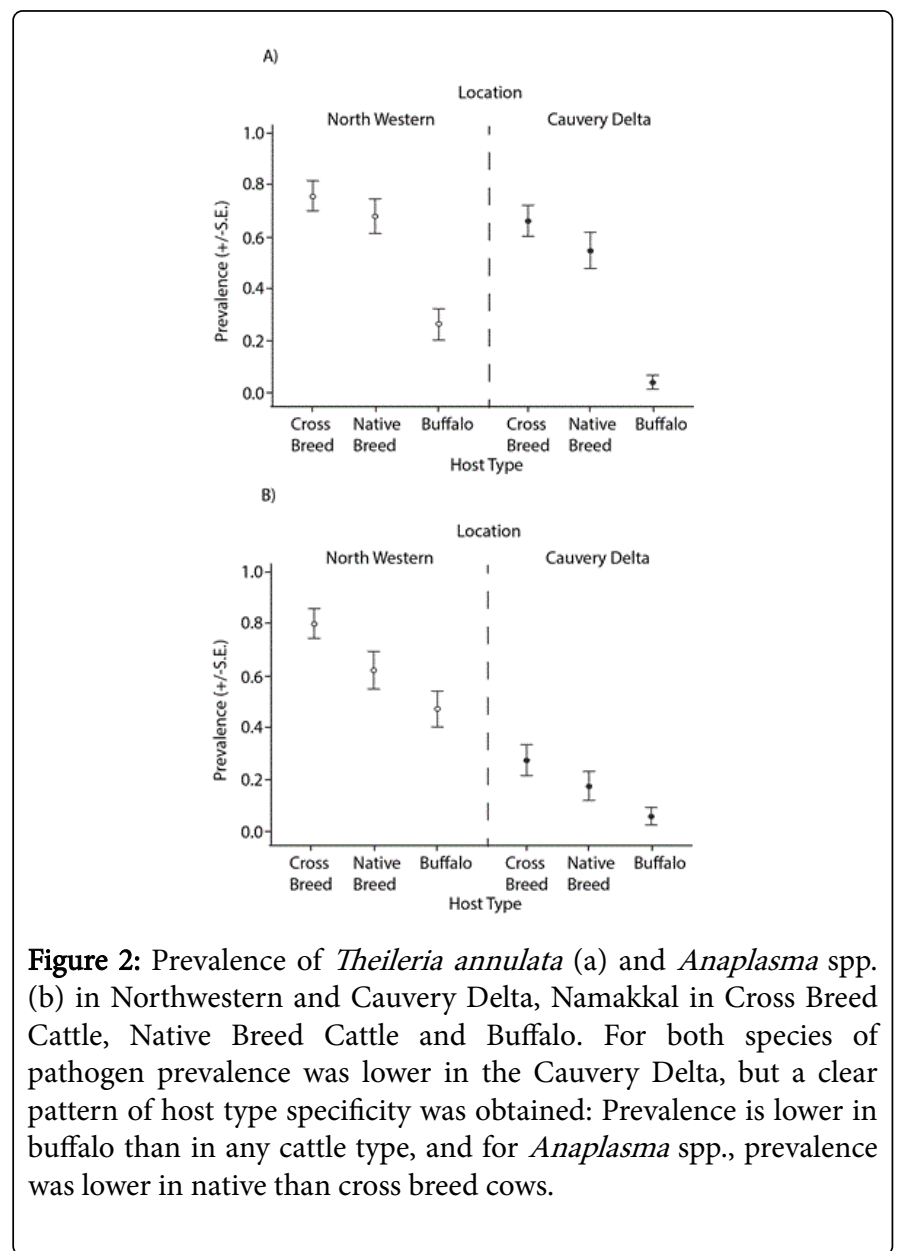




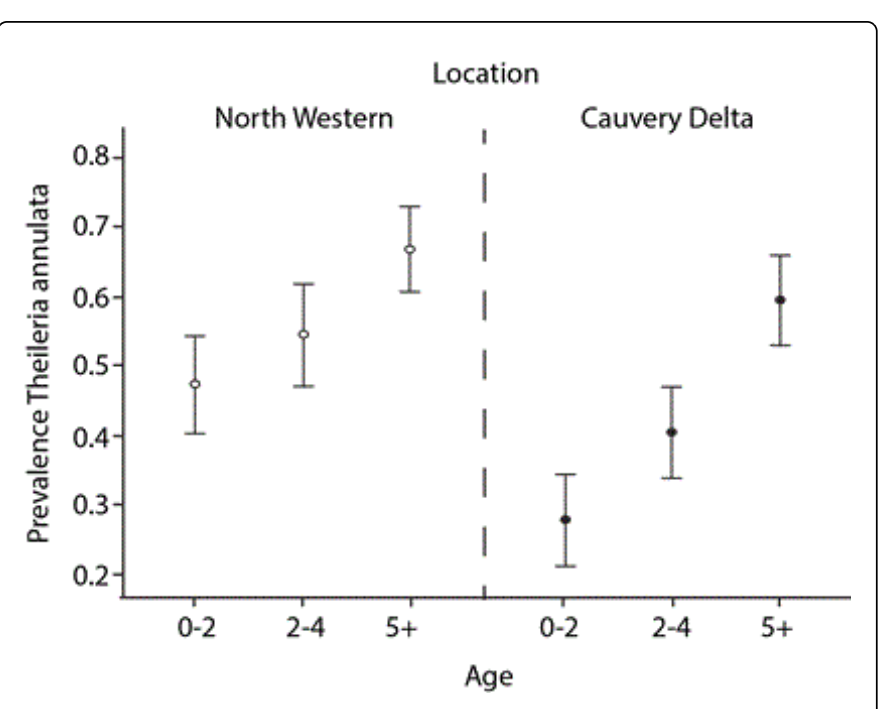

Figure 3: Prevalence of Theileria annulata increases with age in cattle and buffalo in Nammakal. Data plotted by location shows the same trend regardless of overall prevalence mediated by environmental effects.

Thus we simplified tick score to a binary classification of ongoing tick infestation (yes or no) and re-ran the model. As expected, a significant effect was now indicated: animals with tick infestations (of any severity) have a greater prevalence of $T$. annulata infection, with around $30 \%$ more animals infected.

Anaplasma spp: As with Theileria, a significant impact of location on the prevalence of Anaplasma spp. was found, so GLMMs using location as a random effect were fitted for testing fixed factors. We found a significant effect of host type and tick infestation on the prevalence of Anaplasma. Unlike $T$. annulata there was no significant effect of age (Table 2). Significantly lower prevalence in buffalo than in cattle was detected. In addition, a significant difference in prevalence between native and crossbreed cattle (LSMeans $\mathrm{t}=-2.96, \mathrm{p}=0.003$ ) was found for Anaplasma, with significantly fewer infected native breed than crossbreed animals (Figure 2). Animals without on going tick infestations had a significantly lower prevalence of Anaplasma spp. than those with ticks, though in this case it is important to stress that the differences in prevalence between breed types for cattle were not mediated by tick exposure, since there was no difference in infestation between cattle breed-type (see above). Therefore, the prevalence of TBP in bovine animals in this study is a most likely a consequence of both extrinsic (exposure to ticks) and intrinsic (genetic resistance mediated by host type) effects. We found a significant impact of sex on prevalence of Anaplasma, this result should be treated with caution: male crossbreed cows and buffalos are not kept on farms, as they are considered uneconomic, while male native breeds are kept for labour purposes (ploughing/draughting etc). Therefore all males in the study were native breeds, completely blocking assessment of sex within host type, and when considered in natives alone there was no difference in Anaplamsa prevalence in male and female cattle $(\mathrm{p}>0.9$, mean prevalence: males $=0.39$, females $=0.41$ ).

\section{Discussion}

This study set out to empirically test whether the prevalence of some tick-borne infections of bovines in an important region of dairy production in India is altered by regional or breed specific differences, with emphasis on the level of carrier state between host types. Such information may be of relevance if one host type over another showed altered levels of carrier state (prevalence and parasitaemia), in the face of similar levels of tick challenge, as this could reduce or exacerbate the infection risk for more susceptible, but more productive breeds. We found good evidence that TBP prevalence levels on farms in Namakkal, India are shaped by a combination of intrinsic and extrinsic effects. Importantly, the prevalence of carrier status of Theileria annulata and Anaplasma spp. were indicated as dependent on host type and, not surprisingly, tick exposure.

In general, the overall prevalence of $T$. annulata, except in buffaloes $(15.5 \%)$, was above $60 \%$, which is threefold higher than the prevalence rate of T. annulata (19\%) recorded by others in the Indian subcontinent [17,18]. The prevalence of Anaplasma spp was also higher than other PCR surveys in South India [19]. These high prevalence values were only detected using the PCR based assay. Only 11 animals (3.3\%) in our study were diagnosed positive for piroplasms of Theileria sp by blood smear examination; a finding in agreement with some related surveys $[20,21]$ but not others [22]. Together our results show that to accurately estimate the reservoir of infection and assess factors that could influence the risk of new infections occurring, PCR is a more sensitive technique [23].

It has been suggested that the presence of animals less susceptible to becoming reservoirs of transmittable infection in a population may have a diluting effect on levels of tick-borne disease in the population as a whole [9]. Our data supports the idea that buffalo and native breeds of cattle are more resistant to becoming carrier animals for some TBP than crossbred cattle. For both Anaplasma spp and Theileria annulata, buffalo were less likely than either species of cattle to have carrier status infections. If the force of infection for both parasites is identical across host types this would lend support to the idea that cograzing of crossbreeds and buffalo could lead to a dilution effect for tick challenge: buffalo reducing subsequent infection risk by having a lower probability of acting as a reservoir for tick transmission. However, the results showed strong evidence that buffalo are less likely than cattle to have detectable tick infestations. The reason for this difference is unclear, but of potential importance to dairy farming in the area. Possible hypotheses for this difference are: buffalo are just as attractive to questing ticks but ticks are more likely to drop off before repletion; buffalo repel ticks prior to infestation; or ticks prefer to feed on co-grazed cross-breed cows. There is some evidence that intrinsic differences in tick susceptibility can be mediated by a combination of such effects in the context of indicus and taurine African cattle breeds [24], but elucidating the most important proximate mechanisms is of special importance in the Indian context: If ticks that fail to feed on buffalo subsequently quest for an alternative host then co grazing would not reduce challenge of cattle and, theoretically, may increase it. Further investigation of subsequent questing behaviour of ticks that fail to feed on buffalo is warranted to determine if buffalo exacerbate, diminish or are neutral with respect to tick challenge of co-grazed cattle. In addition it seems clear that while buffalo could excaerbate the risk of tick challenge they do not appear to provide a substantial reservoir of parasite challenge for cattle. Thus, the epidemiology of $T$. annulata may not mirror that of $T$. parva where buffalo act to provide a large reservoir of parasite genotypes that can trasmit to cattle and cause disease [25,26]. Comparative investigation of genotype complexity of $T$. annulata derived from buffalo and cattle is required to support this theory. 
Page 6 of 7

The detected difference in prevalence of Anaplasma spp. between native and crossbreed cattle is likely to be determined partly by host genetic resistance: we found no evidence of a difference in tick exposure between cattle breed types but around $10 \%$ difference in Anaplasma spp. prevalence. Evidence of a difference in cattle breedtype specific outcome of infection is also provided by the comparison of blood smears to PCR for detection of T. annulata. Only cross breed cattle scored positive by blood smear the logical conclusion being that this relates to a higher parasitaemia in crossbreed animals. Whether lower levels of pathogen burden are linked to genetic resistance of natives vs cross breeds requires to be established, although is indicated from previous studies $[27,28]$. Mechanisms of breed resistance against T. annulata are known to be manifest as a reduction in clinical paramaters associated with an alteraltion in the level of proinflammatory cytokines [29]. A reduced pathogen burden would indicate either reduced ability of the infection to amplify and/or a greater ability of resistant animals to control the infection. Whether a lower parasitaemia means that natives are less of a risk for transmission of $T$. annulata than crossbreeds requires investigation. In terms of general prevalence, our results indicate that native cattle will act as a common reservoir for transmission of infection to crossbreeds when co-grazed.

Substantial differences in the prevalence of $T$. annulata, and especially Anaplasma sp, were found between the two regions in this study. This could be due to differences in climatic and geographical variations between study sites that alter vector abundance or competency. There was some evidence that the species composition of ticks found might have differed between regions: Haemaphysalis intermedia and Rhicephalus were identified in greater numbers in the North Western zone. However, given that their abundance was low, and neither is considered a principle vector for T. annulata, it seems unlikely that these species were major contributors to geographic prevalence. A clear age related pattern in the prevalence of Tannulata was detected. Prevalence was higher as the age of animals increased, in contrast to the case for T. parva [30] or T. annulata in Pakistan [31]. The higher prevalence in adults could be due to stress associated (parturition and transport) immunosuppression, or indicate that the level of infected tick challenge is not sufficient to infect all calves [32]. Adult animals may also be more likely to be exposed to tick infestation when they are grazed on community pasture. This result raises questions about the best management practice for older cows, especially those that are past peak productivity. If older cows with higher prevalence of disease act as a reservoir of infection for younger cows, co-grazing different age groups may be detrimental to milk yield overall [33].

In conclusion, the results of this study indicate the importance of host type in determining patterns of parasite prevalence and resistance to clinical disease in dairy herds. There is a need to determine whether crossbreeds pose a greater risk for transmission of disease than native cattle, with the role of co-grazing buffalo in influencing this risk also requiring further assessment. As the cost of carrier infection is indicated to be higher in crossbreed cattle, control strategies should be aimed at either improving innate resistance to infection, while retaining productivity, or blocking pathogen transmission.

\section{Acknowledgements}

The authors would like to thank veterinarians who had rendered their unstinted help during sample collections and also thank the Dean, Veterinary College and Research Institute, Namakkal, for facilities provided. This project was funded by DBT, Govt. of India under Indo-UK (BBSRC) collaborative project grant number $\mathrm{BB} /$ L004739/1.

\section{References}

1. USDA Foreign Agricultural Service (2016) Global Agricultural Information Network. Report no IN6126.

2. Neitz WO (1957) Theileriosis, gonderiosis and Cytauxzooses: a review. Onderstepoort J Vet Res 27: 275-430.

3. Velusamy R, Rani N, Ponnudurai G, Harikrishnan J, Anna T, et al. (2014) Influence of season, age and breed on prevalence of haemoprotozoan diseases in cattle of Tamil Nadu, India. Vet World 7: 574-578.

4. Devendra C (1995) In: Global agenda for Livestock Research, EDS, ILRI, Nairobi pp: 41-48.

5. Gharbi M, Touay A, Khayeche M, Laarif J, Jedidi M, et al. (2011) Ranking control options for tropical theileriosis in at-risk dairy cattle in Tunisia, using benefit-cost analysis. Rev Sci Tech 30: 763-778.

6. Kolte SW, Larcombe SD, Jadhow SG, Magar SP, Warthi G, et al. (2017) PCR diagnosis of tick-borne pathogens in Maharashtra state, India indicates fitness cost associated with carrier infections is greater for crossbreed than native cattle breeds.

7. Graham OH, Hourrigan JL (1977) Review article: Eradication programs for the arthropod parasites of livestock. J Med Entomol 13: 629-658.

8. George JE, Pound JM, Davey RB (2004) Chemical control of ticks on cattle and the resistance of these parasites to acaricides. Parasitol 129: S353-S366.

9. Schmidt KA, Ostfeld RS (2001) Biodiversity and the dilution effect in disease ecology Ecology 82: 609-619.

10. Hoogstral H (1956) African Ixodoidea I, ticks of Sudan,. Research Report No. NM 005.29.07. US Dept of Navy, Bureau of Medicine and Surgery, Washington DC.

11. Sen SK, Fletcher TP (1962) Veterinary Parasitology and Acarology for India. Indian Council of Agricultural Research pp: 586-612.

12. Walker JB, Keirans JE, Horok IG (2000) The genus Rhipicephalus (Acari, Ixodidae): A guide to the brown ticks of the world. Cambridge University Press pp: 520-521.

13. Julong L, Li Y, Liu A, Guan G, Xie J (2015) Development of a multiplex PCR assay for detection and characterization of Theileria annulata and Theileria sergenti in cattle. Parasitol Res 114: 2715-2721.

14. Schabenberger O (2011) The GLIMMIX procedure using SAS USA.

15. Noaman V (2014) Comparison of molecular and microscopic technique for detection of Theileria spp. in carrier cattle. J Parasit Dis 38: 64-67.

16. Chauhan HC, Patel BK, Bhagat AG, Patel MV, Patel SI (2015) Comparison of molecular and microscopic technique for detection of Theileria annulata from the field cases of cattle. Vet World 8: 1370-1374.

17. Dumanli N, Aktas M, Cetinkala B, Cakmak A, Koroglu E (2005) Prevalence and distribution of tropical theileriosis in eastern Turkey. Vet Parasitol 127: 9-15.

18. Shahnawaz S, Ali M, Aslam MA, Fatima R, Chaudhry ZI (2011) A study on the prevalence of a tick transmitted pathogen, Theileria annulata, and hematological profile of cattle from Southern Punjab. Parasitol Res 109: 1155-1160.

19. Nair AS, Ravindran R, Lakshmanan B, Sreekumar C, Kumar SS (2013) Bovine carriers of Anaplasma marginale and Anaplasma bovis in South India. Trop Biomed 30: 105-112.

20. Durani AZ, Mehmood N, Shakoori AR (2010) Comparison of three diagnostic methods for Theileria annulata in Sahiwal and Friesian cattle in Pakistan. Pakistan J Zool 42: 467-472.

21. Hoghooghi-Rad N, Ghaemi P, Shayan P, Eckert B, Sadr-Shirazi N (2011) Detection of native carrier cattle infected with Theileria annulata by semiNested PCR and Smear method in Golestan Province of Iran, World. Appl Sci J 12: 317-323. 
Citation: Ponnudurai G, Larcombe S, Velusamy R, Rani N, Kolte SW, et al. (2017) Prevalence of Tick-borne Pathogens in Co-grazed Dairy Bovines Differs by Region and Host-type in Tamil Nadu, India. J Adv Dairy Res 5: 177. doi:10.4172/2329-888X.1000177

Page 7 of 7

22. Singh NK, Singh H, Jyoti, Haque M, Rath SS (2012) Prevalence of parasitic infections in cattle of Ludhiana district, Punjab. J Parasit Dis 36: 256-259.

23. Mohamed AM, Abdel-Rady A, Ahmed LS, El-Hosary A (2010) Field evaluation of PCR assay for diagnosis of tropical Theileriosis in cattle and water buffaloes in Egypt. J Anim Vet Adv 9: 696-699.

24. Mattioli RC, Pandey VS, Murray M, Fitzpatrick JL (2000) Immunogenetic influences on tick resistance in African cattle with particular reference to trypanotolerant N'Dama (Bos taurus) and trypanosusceptible Gobra zebu (Bos indicus) cattle. Acta Tropica 75: 263-277.

25. Sitt T, Poole EJ, Ndambuki G, Mwaura S, Njoroge T (2015) Exposure of vaccinated and naive cattle to natural challenge from buffalo-derived Theileria parva. Int J Parasitol 4: 244-251.

26. Rukambile E, Machuka E, Njahira M, Kyalo M, Skilton R (2016) Population genetic analysis of Theileria parva isolated in cattle and buffaloes in Tanzania using minisatellite and microsatellite markers. Vet Parasitol 224: 20-26.

27. Glass EJ, Preston PM, Springbett A, Craigmile S, Kirvar E (2005) Bos taurus and Bos indicus (Sahiwal) calves respond differently to infection with Theileria annulata and produce markedly different levels of acute phase proteins. Int J Parasitol 35: 337-347.
28. Bock RE, de Vos AJ, Kingston TG, McLellan DJ (1997) Effect of breed of cattle on innate resistance to infection with Babesia bovis, B bigemina and Anaplasma marginale. Aust Vet J 75: 337-340.

29. Glass E, Jensen K (2007) Resistance and susceptibility to a protozoan parasite of cattle-Gene expression differences in macrophages from different breeds of cattle. Vet Immunol Immunopath 120: 20-30.

30. Salih DA, El Hussein AM, Seitzer U, Ahmed JS (2007) Epidemiological studies on tick borne diseases of cattle in central Equatoria state, Southern Sudan. Parasitol Res 101: 1035-1044.

31. Qayyum M, Farooq U, Samad HA, Chauhdry HR (2010) Prevalence, Clinicotherapeutic and prophylactic studies on theileriosis in district Sahiwal. J Anim Plant Sci 20: 266-270.

32. Magona JW, Walubengo J, Olaho-Mukani W, Jonsson NN, Welburn SW (2011) Spatial variation of tick abundance and seroconversion rates of indigenous cattle to Anaplasma marginale, Babesia bigemina and Theileria parva infections in Uganda. Exp App Acarol 55: 203-213.

33. Brown CG (1990) Control of tropical theileriosis (Theileria annulata infection) of cattle. Parasitologia 32: 23-31. 